\title{
External approximation of nonlinear operator equations
}

\author{
Etienne Emmrich ${ }^{\mathrm{a}}$ \\ ${ }^{a}$ TU Berlin, Institut für Mathematik, Straße des 17. Juni 136, 10623 Berlin \\ (January 4, 2009)
}

\begin{abstract}
Based upon an external approximation scheme for the underlying Banach space, a nonlinear operator equation is approximated by a sequence of coercive problems. The equation is supposed to be governed by the sum of two nonlinear operators acting between a reflexive Banach space and its dual. Under suitable stability assumptions and if the underlying operators can be approximated consistently, weak convergence of a subsequence of approximate solutions is shown. This also proves existence of solutions to the original equation.
\end{abstract}

Keywords: Nonlinear operator equation; monotone operator; perturbation; external approximation scheme; convergence

AMS Subject Classification: 65J15; 65N12; 47J25; 47H05

\section{Introduction}

Let $V$ be a real reflexive Banach space with its dual $V^{*}$. We are concerned with the approximate solution of the following problem: For $f \in V^{*}$ find $u \in V$ such that

$$
A u+B u=f \quad \text { in } V^{*} .
$$

Here, $A: V \rightarrow V^{*}$ and $B: V \rightarrow V^{*}$ are given operators. A standard situation is $A$ being monotone and hemicontinuous, $B$ being a strongly continuous perturbation of $A$ and $A+B$ being coercive.

Many boundary value problems for quasilinear partial differential equations arising in physics, fluid mechanics and other areas of application can be formulated as (1) (see, e.g., the monographs $[6,8,19]$ and the references therein). For their approximate solution, often Galerkin-type or finite difference methods are employed. The study of all these methods can be unified by considering so-called external approximation schemes. This concept covers in particular finite differences, nonconforming finite elements as well as fully discrete finite element methods with quadrature (see $[15,16]$ for several examples).

External approximation schemes have been studied in [13] in the context of the convergence of finite difference approximations for quasilinear partial differential equations and A-proper operators (see [16], [19, Ch. 35] for introductions into the concept of external approximation schemes). Later on, extensions of the results obtained in [13] have been studied in $[17,18]$, the focus being on the equivalence of unique solvability of the original and approximate problem.

External approximations have also been studied for eigenvalue problems and the phenomenon of superconvergence (see [10-12]) and, more recently, for the approx- 
imation of variational problems in spaces of piecewise constant functions (see [5]). The concept of external approximation has further been employed in [15] for studying different numerical methods for solving the Navier-Stokes problem that is, in the stationary case, indeed of the type (1) with $A$ being linear and strongly positive, $B$ being strongly continuous and $A+B$ being coercive.

The assumptions on the approximating operators studied so far in $[13,17,18]$ (in particular, an inverse stability that would follow from stronger uniform monotonicity assumptions) are, however, different from the assumptions on which our studies are based. Indeed, we try to apply the concept of external approximation in order to generalise the often found standard situation for (1) with $A$ being monotone and hemicontinuous, $B$ being strongly continuous and $A+B$ being coercive. It is well-known that in this situation Brézis' theorem on pseudomonotone operators provides existence of solutions to (1). So, our approach is not based upon the concept of A-properness (see [9] and the references cited therein for a discussion of this concept) but on weaker assumptions yielding then only convergence of a subsequence in a weak sense. In particular, we do not assume well-posedness of the approximate problems but only solvability and, in general, we do not have a continuous inverse of the approximate operator (for this case, see also the exhaustive work [3]).

Another approach for studying approximations of monotone operator equations employs projection methods as in $[1,2,6]$, which can be interpreted as internal approximation schemes. The essential advantage of external approximation schemes, however, is that the function spaces approximating $V$ need not to be subspaces of $V$. This allows much more flexibility in the choice of the numerical method and often simplifies the numerical analysis.

We should mention that, besides the concept of external approximation, also the concept of discrete convergence and discrete approximation as introduced in [14], which goes without prolongation and restriction operators, provides a frame for considering rather different numerical methods in a unifying way. This concept has been applied in [7] to the study of nonlinear operator equations based upon the notion of approximation-regular operators, which is a generalisation of A-properness. The results in [7] also cover quasilinear elliptic problems (leading to a coercive and strictly monotone operator equation of the type (1)) under perturbation of the domain or coefficients.

The main result in this paper will be a general convergence result for the case that there is a stable and admissible external approximation scheme and that $A, B, f$ in (1) can be approximated in a consistent way. The required assumption on the sequence of approximations of $A$ can be seen as a discrete analogue of the property $(\mathrm{M})$. Moreover, our convergence result is based upon an a priori estimate for the sequence of approximate solutions which follows from uniform coercivity. The Galerkin method in the standard situation as described above is shown to be a special case of our approximation scheme.

The paper is organised as follows: In Section 2, we describe the external approximation scheme, present the necessary notation, and prove some results on the consistency and solvability of the approximation. The main result is stated and proven in Section 3. Section 4 provides an example.

\section{The external approximation scheme}

For a normed space $X$, we always denote its norm by $\|\cdot\|_{X}$, its dual space by $X^{*}$ with standard norm $\|\cdot\|_{X^{*}}$ and the dual pairing by $\langle\cdot, \cdot\rangle$.

Let $V$ be a given real reflexive Banach space. Let $\mathscr{H}$ be a countable infinite 
sequence of indices and let $\left\{\left(V_{h}, p_{h}, r_{h}\right)\right\}_{h \in \mathscr{H}}$ be a sequence of real normed spaces $V_{h}$, prolongation operators $p_{h}: V_{h} \rightarrow F$ and restriction operators $r_{h}: V \rightarrow V_{h}$. The prolongation operators are assumed to be linear and bounded. Here, $F$ is a suitably chosen real reflexive Banach space such that there is a so-called synchronisation operator $\omega: V \rightarrow F$ that is linear, bounded and injective. The family $\left\{\left(V_{h}, p_{h}, r_{h}\right)\right\}_{h \in \mathscr{H}}$ then is said to be an external approximation scheme for $V$. In some situations, the restriction operators are only defined on a dense subset of $V$ but can be extended on $V$ (see [15, Prop. 3.1 on p. 30], [16, Prop. 4 on p. 28]).

Definition 2.1: An external approximation scheme $\left\{\left(V_{h}, p_{h}, r_{h}\right)\right\}_{h \in \mathscr{H}}$ for $V$ is said to be stable iff there is a constant $c>0$ such that for all $h \in \mathscr{H}$

$$
\left\|p_{h} v_{h}\right\|_{F} \leq c\left\|v_{h}\right\|_{V_{h}} \quad \forall v_{h} \in V_{h} .
$$

It is said to be admissible iff it fulfills

(i) the compatibility condition:

$$
p_{h} r_{h} v \rightarrow \omega v \text { in } F(h \in \mathscr{H}) \quad \forall v \in V,
$$

(ii) the synchronisation condition: for any subsequence $\mathscr{H}^{\prime} \subseteq \mathscr{H}$ of indices and $\left\{v_{h}\right\}_{h \in \mathscr{H}^{\prime}} \in\left\{V_{h}\right\}_{h \in \mathscr{H}^{\prime}}, g \in F$ with $p_{h} v_{h} \rightarrow g$ in $F\left(h \in \mathscr{H}^{\prime}\right)$ there is an element $v \in V$ such that $\omega v=g$.

Note that the use of the foregoing notions is not consistent in the literature. We now consider the sequence of approximate problems: For $f_{h} \in V_{h}^{*}$ find $u_{h} \in V_{h}$ such that

$$
A_{h} u_{h}+B_{h} u_{h}=f_{h} \quad \text { in } V_{h}^{*} .
$$

Here, $\left\{A_{h}\right\}_{h \in \mathscr{H}},\left\{B_{h}\right\}_{h \in \mathscr{H}}$ and $\left\{f_{h}\right\}_{h \in \mathscr{H}}$ are sequences of operators and functionals approximating $A, B$ and $f$, respectively.

Definition 2.2: Let $\left\{\left(V_{h}, p_{h}, r_{h}\right)\right\}_{h \in \mathscr{H}}$ be a stable and admissible external approximation scheme for $V$. A sequence $\left\{\left(A_{h}, B_{h}, f_{h}\right)\right\}_{h \in \mathscr{H}}$ of operators $A_{h}: V_{h} \rightarrow$ $V_{h}^{*}, B_{h}: V_{h} \rightarrow V_{h}^{*}$ and functionals $f_{h} \in V_{h}^{*}$ is said to be a consistent approximation of $(A, B, f)$ iff for any subsequence $\mathscr{H}^{\prime} \subseteq \mathscr{H}$ and any $\left\{v_{h}\right\}_{h \in \mathscr{H}^{\prime}} \in\left\{V_{h}\right\}_{h \in \mathscr{H}^{\prime}}$, $v \in V$ with $p_{h} v_{h} \rightarrow \omega v$ in $F\left(h \in \mathscr{H}^{\prime}\right)$ there holds

(i) if there is an element $g \in F$ such that

$$
\left\langle A_{h} v_{h}, r_{h} w\right\rangle \rightarrow\langle g, w\rangle \quad \forall w \in V, \quad \limsup _{h \in \mathscr{H}^{\prime}}\left\langle A_{h} v_{h}, v_{h}\right\rangle \leq\langle g, v\rangle
$$

then $A v=g$ in $V^{*}$;

$$
\begin{aligned}
& \left\langle B_{h} v_{h}, r_{h} w\right\rangle \rightarrow\langle B v, w\rangle \quad \forall w \in V, \quad \liminf _{h \in \mathscr{H}^{\prime}}\left\langle B_{h} v_{h}, v_{h}\right\rangle \geq\langle B v, v\rangle ; \\
& \left\langle f_{h}, r_{h} w\right\rangle \rightarrow\langle f, w\rangle \quad \forall w \in V, \quad \limsup _{h \in \mathscr{H}^{\prime}}\left\langle f_{h}, v_{h}\right\rangle \leq\langle f, v\rangle .
\end{aligned}
$$

We remark that condition (i) in the foregoing definition is a discrete counterpart of the property (M) (see [8, p. 173]). A standard example is given by

Proposition 2.3: Let $\left\{V_{h}\right\}_{h \in \mathscr{H}}$ be a Galerkin scheme for $V, p_{h}: V_{h} \rightarrow V$ be the identity and $r_{h}: V \rightarrow V_{h}$ such that $r_{h} v$ is a best approximation of $v \in V$ in $V_{h}$. Then $\left\{\left(V_{h}, p_{h}, r_{h}\right)\right\}_{h \in \mathscr{H}}$ is a stable and admissible external approximation scheme with $F=V$. Let $A_{h}=p_{h}^{*} A p_{h}$ (with $p_{h}^{*}: V^{*} \rightarrow V_{h}^{*}$ denoting the dual operator of $\left.p_{h}\right), B_{h}=p_{h}^{*} B p_{h}$ and $f_{h}=p_{h}^{*} f$. If $A: V \rightarrow V^{*}$ is monotone and 
hemicontinuous and $B: V \rightarrow V^{*}$ is strongly continuous then $\left(A_{h}, B_{h}, f_{h}\right)_{h \in \mathscr{H}}$ is a consistent approximation of $(A, B, f)$.

Proof: Stability and admissibility of the external approximation scheme built by a Galerkin scheme is evident. For proving consistency, let $\mathscr{H}^{\prime} \subseteq \mathscr{H}$ and $\left\{v_{h}\right\}_{h \in \mathscr{H}^{\prime}} \in$ $\left\{V_{h}\right\}_{h \in \mathscr{H}^{\prime}}, v \in V$ with $p_{h} v_{h} \rightarrow \omega v=v$ in $F=V\left(h \in \mathscr{H}^{\prime}\right)$ be given. In what follows, all convergence is meant for $h \in \mathscr{H}^{\prime}$.

(i) With $A_{h}=p_{h}^{*} A p_{h}$, we find from the definition of the dual operator

$$
\left\langle A_{h} v_{h}, w_{h}\right\rangle=\left\langle A p_{h} v_{h}, p_{h} w_{h}\right\rangle \quad \forall w_{h} \in V_{h} .
$$

Let $w \in V$ be arbitrary. The monotonicity of $A: V \rightarrow V^{*}$ then provides

$$
\begin{aligned}
\left\langle A_{h} v_{h}, v_{h}\right\rangle & =\left\langle A p_{h} v_{h}, p_{h} v_{h}\right\rangle \\
& \geq\left\langle A p_{h} v_{h}, p_{h} v_{h}\right\rangle-\left\langle A p_{h} v_{h}-A w, p_{h} v_{h}-w\right\rangle \\
& =\left\langle A w, p_{h} v_{h}-w\right\rangle+\left\langle A p_{h} v_{h}, p_{h} r_{h} w\right\rangle+\left\langle A p_{h} v_{h}, w-p_{h} r_{h} w\right\rangle .
\end{aligned}
$$

The first term on the right-hand side of (3) converges towards $\langle A w, v-w\rangle$ since $p_{h} v_{h} \rightarrow v$ in $V$. The second term $\left\langle A p_{h} v_{h}, p_{h} r_{h} w\right\rangle=\left\langle A_{h} v_{h}, r_{h} w\right\rangle$ converges towards $\langle g, w\rangle$ by the assumption in Definition 2.2 (i). For the third term, we observe that $\left\{A p_{h} v_{h}\right\}_{h \in \mathscr{H}^{\prime}}$ is bounded in $V^{*}$, which follows (see, e.g., [6, Folg. 1.2 on p. 65]) from the boundedness of $\left\{\left\langle A p_{h} v_{h}, p_{h} v_{h}\right\rangle\right\}_{h \in \mathscr{H}^{\prime}}$ (by the assumption in Definition 2.2 (i)), the boundedness of $\left\{p_{h} v_{h}\right\}_{h \in \mathscr{H}^{\prime}}$ in $V$ (the sequence is weakly convergent) and the monotonicity of $A: V \rightarrow V^{*}$. The third term thus vanishes in the limit since $p_{h} r_{h} w \rightarrow w$ in $V$ (compatibility). In the limit, we finally obtain from (3) together with the assumption in Definition 2.2 (i)

$$
\begin{aligned}
\langle g, v\rangle & \geq \limsup _{h \in \mathscr{H}^{\prime}}\left\langle A_{h} v_{h}, v_{h}\right\rangle \\
& \geq \lim _{h \in \mathscr{H}^{\prime}}\left(\left\langle A w, p_{h} v_{h}-w\right\rangle+\left\langle A p_{h} v_{h}, p_{h} r_{h} w\right\rangle+\left\langle A p_{h} v_{h}, w-p_{h} r_{h} w\right\rangle\right) \\
& =\langle A w, v-w\rangle+\langle g, w\rangle .
\end{aligned}
$$

Taking $w=v \pm s z$ for arbitrary $z \in V$ and $s \in(0,1]$ yields

$$
\langle A(v+s z), z\rangle \geq\langle g, z\rangle \text { and }\langle A(v-s z), z\rangle \leq\langle g, z\rangle,
$$

and with $s \rightarrow 0+$, the hemicontinuity of $A: V \rightarrow V^{*}$ shows $\langle A v, z\rangle=\langle g, z\rangle$ and hence $A v=g$.

(ii) With $B_{h}=p_{h}^{*} B p_{h}$, we find for all $w \in V$

$$
\left\langle B_{h} v_{h}, r_{h} w\right\rangle=\left\langle B p_{h} v_{h}, p_{h} r_{h} w\right\rangle
$$

Since $B: V \rightarrow V^{*}$ is strongly continuous and $p_{h} v_{h} \rightarrow v$ in $V$, it follows $B p_{h} v_{h} \rightarrow B v$ in $V^{*}$. Because of $p_{h} r_{h} w \rightarrow w$ in $V$, we come up with

$$
\left\langle B_{h} v_{h}, r_{h} w\right\rangle \rightarrow\langle B v, w\rangle
$$

Moreover, we have

$$
\left\langle B_{h} v_{h}, v_{h}\right\rangle=\left\langle B p_{h} v_{h}, p_{h} v_{h}\right\rangle \rightarrow\langle B v, v\rangle .
$$


(iii) With respect to the approximation of $f \in V^{*}$, we observe for all $w \in V$

$$
\left\langle f_{h}, r_{h} w\right\rangle=\left\langle f, p_{h} r_{h} w\right\rangle \rightarrow\langle f, w\rangle
$$

as well as (remember $p_{h} v_{h} \rightarrow v$ in $V$ )

$$
\left\langle f_{h}, v_{h}\right\rangle=\left\langle f, p_{h} v_{h}\right\rangle \rightarrow\langle f, v\rangle .
$$

Note that a best approximation $r_{h} v$ of $v \in V$ in $V_{h}$ always exists if $\operatorname{dim} V_{h}<\infty$ but $r_{h}$ might be nonlinear. Instead of the best approximation, one may also take a suitable projection. In [16, p. 28], the restriction operator on $V$ is constructed from its definition on the dense subset $\bigcup_{h \in \mathscr{H}} V_{h}$.

It arises the question, from which assumptions one can derive condition (i) in Definition 2.2. An answer is given by

Proposition 2.4: Let $\left\{\left(V_{h}, p_{h}, r_{h}\right)\right\}_{h \in \mathscr{H}}$ be a stable and admissible external approximation scheme for $V$ and let $A: V \rightarrow V^{*}$ be hemicontinuous. Assume that all $A_{h}: V_{h} \rightarrow V_{h}^{*}(h \in \mathscr{H})$ are monotone and that for any subsequence $\mathscr{H}^{\prime} \subseteq \mathscr{H}$ and any $\left\{v_{h}\right\}_{h \in \mathscr{H}^{\prime}} \in\left\{V_{h}\right\}_{h \in \mathscr{H}^{\prime}}, v \in V$ with $p_{h} v_{h} \rightarrow \omega v$ in $F\left(h \in \mathscr{H}^{\prime}\right)$

$$
\limsup _{h \in \mathscr{H}^{\prime}}\left\langle A_{h} r_{h} w, v_{h}\right\rangle \geq\langle A w, v\rangle \quad \forall w \in V .
$$

Then condition (i) in Definition 2.2 is fulfilled.

Proof: The monotonicity of $A_{h}\left(h \in \mathscr{H}^{\prime}\right)$ yields for arbitrary $w \in V$

$$
\begin{aligned}
\left\langle A_{h} v_{h}, v_{h}\right\rangle & \geq\left\langle A_{h} v_{h}, v_{h}\right\rangle-\left\langle A_{h} v_{h}-A_{h} r_{h} w, v_{h}-r_{h} w\right\rangle \\
& =\left\langle A_{h} r_{h} w, v_{h}-r_{h} w\right\rangle+\left\langle A_{h} v_{h}, r_{h} w\right\rangle .
\end{aligned}
$$

In the limit, we thus obtain by the assumption in Definition 2.2 (i) and with (5)

$$
\begin{aligned}
\langle g, v\rangle & \geq \limsup _{h \in \mathscr{H}^{\prime}}\left\langle A_{h} v_{h}, v_{h}\right\rangle \\
& \geq \limsup _{h \in \mathscr{H}^{\prime}}\left\langle A_{h} r_{h} w, v_{h}-r_{h} w\right\rangle+\lim _{h \in \mathscr{H}^{\prime}}\left\langle A_{h} v_{h}, r_{h} w\right\rangle \\
& \geq\langle A w, v-w\rangle+\langle g, w\rangle .
\end{aligned}
$$

Here, we have employed that $p_{h} v_{h} \rightarrow \omega v, p_{h} r_{h} w \rightarrow \omega w$ in $F\left(h \in \mathscr{H}^{\prime}\right)$. The hemicontinuity of $A$ implies $A v=g$ in $V^{*}$ as in (4).

We will also make use of the following notion.

Definition 2.5: Let $\left\{V_{h}\right\}_{h \in \mathscr{H}}$ be a sequence of normed spaces. A sequence $\left\{T_{h}\right\}_{h \in \mathscr{H}}$ of operators $T_{h}: V_{h} \rightarrow V_{h}^{*}$ is said to be coercive uniformly in $h$ iff there is a function $\gamma: \mathbb{R}_{0}^{+} \rightarrow \mathbb{R}$ with $\gamma(z) \rightarrow \infty$ as $z \rightarrow \infty$ such that for all $h \in \mathscr{H}$

$$
\left\langle T_{h} v_{h}, v_{h}\right\rangle \geq \gamma\left(\left\|v_{h}\right\|_{V_{h}}\right)\left\|v_{h}\right\|_{V_{h}} \quad \forall v_{h} \in V_{h} .
$$

We end this section by presenting a criterion for the existence of solutions to the approximate problem. 
Lemma 2.6: Let $\Phi: \mathbb{R}^{N} \rightarrow \mathbb{R}^{N}$ be continuous. If there is $R>0$ such that $\Phi(\mathbf{v}) \cdot \mathbf{v} \geq 0$ for all $\mathbf{v} \in \mathbb{R}^{N}$ with $\|\mathbf{v}\|_{\mathbb{R}^{N}}=R$ then there exists $\mathbf{u} \in \mathbb{R}^{N}$ with $\|\mathbf{u}\|_{\mathbb{R}^{N}} \leq R$ and $\Phi(\mathbf{u})=0$.

Proof: The proof follows by contradiction from Brouwer's fixed point theorem (see, e.g., [6, Lemma 2.1 on p. 74]).

Theorem 2.7: $\quad$ Let $V_{h}$ be a normed space with $\operatorname{dim} V_{h}=N<\infty$ and let $A_{h}, B_{h}$ : $V_{h} \rightarrow V_{h}^{*}$ be continuous operators such that $A_{h}+B_{h}$ is coercive. For any $f_{h} \in V_{h}^{*}$, equation (2) then possesses a solution.

Proof: Let $\left\{e_{i}\right\}_{i=1}^{N}$ be a basis in $V_{h}$. Then there is a bijective mapping between $V_{h}$ and $\mathbb{R}^{N}$ given by the representation

$$
v_{h}=\sum_{i=1}^{N} v_{i} e_{i} \in V_{h}, \quad \mathbf{v}=\left[v_{1}, \ldots, v_{N}\right] \in \mathbb{R}^{N} .
$$

On $\mathbb{R}^{N}$, we define the norm $\|\mathbf{v}\|_{\mathbb{R}^{N}}:=\left\|v_{h}\right\|_{V_{h}}$ and the mapping $\Phi(\mathbf{v})=\left[\Phi_{1}, \ldots, \Phi_{N}\right]$ with

$$
\Phi_{i}(\mathbf{v}):=\left\langle A_{h} v_{h}+B_{h} v_{h}-f_{h}, e_{i}\right\rangle \quad(i=1, \ldots, N) .
$$

Obviously, $\Phi: \mathbb{R}^{N} \rightarrow \mathbb{R}^{N}$ is continuous if $A_{h}, B_{h}: V_{h} \rightarrow V_{h}$ are continuous.

Because of the coercivity of $A_{h}+B_{h}$, we find (with a function $\gamma: \mathbb{R}_{0}^{+} \rightarrow \mathbb{R}$ with $\gamma(z) \rightarrow \infty$ as $z \rightarrow \infty)$

$$
\Phi(\mathbf{v}) \cdot \mathbf{v}=\left\langle A_{h} v_{h}+B_{h} v_{h}-f_{h}, v_{h}\right\rangle \geq \gamma\left(\left\|v_{h}\right\|_{V_{h}}\right)\left\|v_{h}\right\|_{V_{h}}-\left\|f_{h}\right\|_{V_{h}^{*}}\left\|v_{h}\right\|_{V_{h}} \geq 0
$$

if $\left\|v_{h}\right\|_{V_{h}}=\|\mathbf{v}\|_{\mathbb{R}^{N}}$ is sufficiently large.

Lemma 2.6 now yields the existence of $\mathbf{u} \in \mathbb{R}^{N}$ and thus of $u_{h} \in V_{h}$ such that $\Phi(\mathbf{u})=0$. But then $u_{h}$ solves (2).

In applications, the continuity of the approximate operators in a finite dimensional space often follows already from the hemicontinuity of the operators $A, B$.

\section{Convergence}

The main result can be formulated as follows.

Theorem 3.1: $\quad$ Suppose there is a consistent approximation of $(A, B, f)$. Assume further that (2) possesses a solution $u_{h} \in V_{h}$ for any $h \in \mathscr{H}$, that the operators $A_{h}+B_{h}: V_{h} \rightarrow V_{h}^{*}(h \in \mathscr{H})$ are coercive uniformly in $h$ and that the sequence $\left\{\left\|f_{h}\right\|_{V_{h}^{*}}\right\}_{h \in \mathscr{H}}$ is bounded. Then there is a subsequence $\mathscr{H}^{\prime} \subseteq \mathscr{H}$ and an element $u \in V$ such that

$$
p_{h} u_{h} \rightarrow \omega u \text { in } F\left(h \in \mathscr{H}^{\prime}\right)
$$

the limit $u$ satisfies (1).

Proof: With the coercivity assumption, we immediately find

$$
\gamma\left(\left\|u_{h}\right\|_{V_{h}}\right)\left\|u_{h}\right\|_{V_{h}} \leq\left\langle A_{h} u_{h}+B_{h} u_{h}, u_{h}\right\rangle=\left\langle f_{h}, u_{h}\right\rangle \leq\left\|f_{h}\right\|_{V_{h}^{*}}\left\|u_{h}\right\|_{V_{h}} .
$$


Since $\gamma(z) \rightarrow z$ as $z \rightarrow \infty$ and since $\left\{\left\|f_{h}\right\|_{V_{h}^{*}}\right\}_{h \in \mathscr{H}}$ is bounded, this shows also the boundedness of $\left\{\left\|u_{h}\right\|_{V_{h}}\right\}_{h \in \mathscr{H}}$.

Because of the stability of the external approximation scheme, then also the sequence $\left\{p_{h} u_{h}\right\}_{h \in \mathscr{H}} \subseteq F$ is bounded. In view of the reflexivity of $F$, there is a subsequence $\mathscr{H}^{\prime} \subseteq \mathscr{H}$ such that $\left\{p_{h} u_{h}\right\}_{h \in \mathscr{H}^{\prime}}$ is weakly convergent in $F$ (see, e.g., [4, Thm. III.27]). Together with the synchronisation condition in Definition 2.1 (ii), there is an element $u \in V$ such that

$$
p_{h} u_{h} \rightarrow \omega u \text { in } F\left(h \in \mathscr{H}^{\prime}\right) .
$$

With Definition 2.2 (ii), (iii), we now find for all $w \in V$

$$
\left\langle f_{h}-B_{h} u_{h}, r_{h} w\right\rangle \rightarrow\langle f-B u, w\rangle, \quad \limsup _{h \in \mathscr{H}^{\prime}}\left\langle f_{h}-B_{h} u_{h}, u_{h}\right\rangle \leq\langle f-B u, u\rangle .
$$

With (2), it follows for all $w \in V$

$$
\left\langle A_{h} u_{h}, r_{h} w\right\rangle=\left\langle f_{h}-B_{h} u_{h}, r_{h} w\right\rangle \rightarrow\langle f-B u, w\rangle
$$

as well as

$$
\limsup _{h \in \mathscr{H}^{\prime}}\left\langle A_{h} u_{h}, u_{h}\right\rangle=\limsup _{h \in \mathscr{H}^{\prime}}\left\langle f_{h}-B_{h} u_{h}, u_{h}\right\rangle \leq\langle f-B u, u\rangle .
$$

Definition 2.2 (i) now provides $A u=f-B u$ in $V^{*}$.

\section{Example}

In order to keep the presentation short, we only consider a somewhat simple example: a linear finite element method with quadrature for a one-dimensional quasilinear Dirichlet problem without perturbation such that $B=B_{h} \equiv 0$.

Let $\psi=\psi(x, t):[0,1] \times \mathbb{R}_{0}^{+} \rightarrow \mathbb{R}$ be a given continuous function. We suppose that $t \mapsto \psi(x, t) t$ is monotonically increasing for all $x \in[0,1]$ and that there is a number $p \in(1, \infty)$ and constants $\mu, c>0$ such that

$$
\psi(x, t) t^{2} \geq \mu t^{p}, \quad|\psi(x, t)| \leq c\left(1+t^{p-2}\right) \quad \forall(x, t) \in[0,1] \times \mathbb{R}_{0}^{+} .
$$

This setting covers, e.g., the one-dimensional $p$-Laplacian.

For a given right-hand side $f \in L^{p^{*}}(0,1)\left(1 / p+1 / p^{*}=1\right)$, then consider the problem

$$
-\left(\psi\left(x,\left|u^{\prime}(x)\right|\right) u^{\prime}(x)\right)^{\prime}=f(x) \quad(x \in(0,1)), u(0)=u(1)=0
$$

The weak formulation of (6) leads to the operator equation (1) with the standard Sobolev space $V=W_{0}^{1, p}(0,1)$ with norm $\|v\|_{V}:=\left(\int_{0}^{1}\left|v^{\prime}(x)\right|^{p} d x\right)^{1 / p}$ and an operator $A: V \rightarrow V^{*}$, defined for $v, w \in V$ via

$$
\langle A v, w\rangle:=\int_{0}^{1} \psi\left(x,\left|v^{\prime}(x)\right|\right) v^{\prime}(x) w^{\prime}(x) d x .
$$


The growth condition for $\psi$ ensures that $A$ maps $V$ into $V^{*}$. The hemicontinuity of $A$ is a direct consequence of the continuity of $\psi$. The right-hand side in (1) is the functional $v \mapsto \int_{0}^{1} f(x) v(x) d x$.

We partition [0,1] equidistantly into $M \in \mathbb{N}$ subintervals $\left[x_{i}, x_{i+1}\right]\left(x_{i}=i / M, i=\right.$ $0, \ldots, M)$ of length $h=1 / M$ and employ linear finite elements. When also applying a simple rectangular rule (taking the right value) for the numerical evaluation of the appearing integrals, we end up with a fully discrete approximation of (6) that can be written in the form (2). This finite element method with quadrature is equivalent to a finite difference scheme.

We take $V_{h}$ as the space of grid functions $v_{h}=\left[v_{h, 0}, \ldots, v_{h, M}\right]^{T} \in \mathbb{R}^{M+1}, v_{h, 0}=$ $v_{h, M}=0$, and endow it with the norm

$$
\left\|v_{h}\right\|_{V_{h}}:=\left(h \sum_{i=1}^{M}\left|D_{i}^{-} v_{h}\right|^{p}\right)^{1 / p}
$$

where $D_{i}^{-} v_{h}:=\left(v_{i}-v_{i-1}\right) / h$. The dual $V_{h}^{*}$ can be identified with the $(M-1)$ dimensional space of grid functions $g_{h}=\left[g_{h, 1}, \ldots, g_{h, M-1}\right]^{T} \in \mathbb{R}^{M-1}$ such that $g_{i}=-D_{i}^{+} w_{h}:=-\left(w_{h, i+1}-w_{h, i}\right) / h(i=1, \ldots, M-1)$ for some $w_{h} \in V_{h}$, the dual pairing is given by

$$
\left\langle g_{h}, v_{h}\right\rangle=h \sum_{i=1}^{M-1} g_{h, i} v_{h, i}=h \sum_{i=1}^{M-1} w_{h, i} D_{i}^{-} v_{h}
$$

The prolongation $p_{h} v_{h}$ of $v_{h} \in V_{h}$ is the piecewise linear interpolation of the points $\left(x_{0}, v_{h, 0}\right), \ldots,\left(x_{M}, v_{h, M}\right)$. The restriction is defined via $\left(r_{h} v\right)_{i}:=v\left(x_{i}\right)$ $(i=0, \ldots, M)$ which is well-defined since $W_{0}^{1, p}(0,1)$ is continuously embedded in $\mathscr{C}([0,1])$. It is easy to show that the sequence $\left\{\left(V_{h}, p_{h}, r_{h}\right)\right\}_{M=1 / h \in \mathbb{N}}$ builds a stable and admissible external approximation scheme for $V$ with $F=V$. In particular, we find for all $v_{h} \in V_{h}$

$$
\left\|p_{h} v_{h}\right\|_{V}=\left\|v_{h}\right\|_{V_{h}},
$$

and the compatibility condition, i.e. $p_{h} r_{h} v \rightarrow v$ in $V$, is easily shown by density.

If we would take a simpler prolongation such as the piecewise constant interpolation of the values of $v_{h}$ as well as of their divided differences then we would come up with $\omega v=\left(v, v^{\prime}\right) \in F=L^{p}(0,1) \times L^{p}(0,1)$.

A simple calculation shows for $v_{h}, w_{h} \in V_{h}$

$$
\left\langle A_{h} v_{h}, w_{h}\right\rangle=h \sum_{i=1}^{M} \psi\left(x_{i},\left|D_{i}^{-} v_{h}\right|\right) D_{i}^{-} v_{h} D_{i}^{-} w_{h},
$$

and, loosely written, we have $\left(A_{h} v_{h}\right)_{i}=-D_{i}^{+}\left(\psi\left(x_{i},\left|D_{i}^{-} v_{h}\right|\right) D_{i}^{-} v_{h}\right)$ for $i=$ $1, \ldots, M-1$. The assumptions on $\psi$ allow to prove in particular that $A_{h}$ maps $V_{h}$ into $V_{h}^{*}$ and is monotone.

In view of Theorem 2.7, the discrete problem is solvable: The continuity of $A_{h}$ : $V_{h} \rightarrow V_{h}^{*}$ is a direct consequence of the continuity of $\psi$. The coercivity (uniform 
in $h$ ) follows from

$$
\left\langle A_{h} v_{h}, v_{h}\right\rangle=h \sum_{i=1}^{M} \psi\left(x_{i},\left|D_{i}^{-} v_{h}\right|\right)\left(D_{i}^{-} v_{h}\right)^{2} \geq \mu h \sum_{i=1}^{M}\left|D_{i}^{-} v_{h}\right|^{p}=\mu\left\|v_{h}\right\|_{V_{h}}^{p}, v_{h} \in V_{h}
$$

We now prove condition (5). Let $p_{h} v_{h} \rightarrow v$ in $V$ (for an arbitrary null sequence of mesh sizes $h$ with $1 / h=M \in \mathbb{N}$ ) and let $w \in V$ be arbitrary. We set $D_{i}^{-} w:=$ $\left(w\left(x_{i}\right)-w\left(x_{i-1}\right)\right) / h(i=1, \ldots, M)\left(\right.$ remember $\left.w \in V=W_{0}^{1, p}(0,1) \hookrightarrow \mathscr{C}([0,1])\right)$. A straightforward calculation shows

$$
\begin{aligned}
& \left\langle A_{h} r_{h} w, v_{h}\right\rangle-\langle A w, v\rangle= \\
& \sum_{i=1}^{M} \int_{x_{i-1}}^{x_{i}}\left(\psi\left(x_{i},\left|D_{i}^{-} w\right|\right) D_{i}^{-} w-\psi\left(x,\left|w^{\prime}(x)\right|\right) w^{\prime}(x)\right) d x D_{i}^{-} v_{h} \\
& +\int_{0}^{1} \psi\left(x,\left|w^{\prime}(x)\right|\right) w^{\prime}(x)\left(\left(p_{h} v_{h}\right)^{\prime}(x)-v^{\prime}(x)\right) d x=: a_{1, h}+a_{2, h} .
\end{aligned}
$$

Denoting by $\psi_{h}$ the w.r.t. the first argument piecewise constant approximation of $\psi$ such that $\psi_{h}(x, t)=\psi\left(x_{i}, t\right)$ for $x \in\left(x_{i-1}, x_{i}\right](i=1, \ldots, M)$ and $t \in \mathbb{R}_{0}^{+}$and upon noting that $\left(p_{h} r_{h} w\right)^{\prime}(x)=D_{i}^{-} w$ for $x \in\left(x_{i-1}, x_{i}\right)(i=1, \ldots, M)$, we find for the first term $a_{1, h}$ by applying Hölder's inequality with $1 / p+1 / p^{*}=1$

$$
\begin{aligned}
& \left|a_{1, h}\right| \\
& \leq\left(h \sum_{i=1}^{M}\left|\frac{1}{h} \int_{x_{i-1}}^{x_{i}}\left(\psi\left(x_{i},\left|D_{i}^{-} w\right|\right) D_{i}^{-} w-\psi\left(x,\left|w^{\prime}(x)\right|\right) w^{\prime}(x)\right) d x\right|^{p^{*}}\right)^{1 / p^{*}}\left\|v_{h}\right\|_{V_{h}} \\
& \leq\left(\sum_{i=1}^{M} \int_{x_{i-1}}^{x_{i}}\left|\psi\left(x_{i},\left|D_{i}^{-} w\right|\right) D_{i}^{-} w-\psi\left(x,\left|w^{\prime}(x)\right|\right) w^{\prime}(x)\right|^{p^{*}} d x\right)^{1 / p^{*}}\left\|v_{h}\right\|_{V_{h}} \\
& =\left(\int_{0}^{1}\left|\psi_{h}\left(x,\left|\left(p_{h} r_{h} w\right)^{\prime}(x)\right|\right)\left(p_{h} r_{h} w\right)^{\prime}(x)-\psi\left(x,\left|w^{\prime}(x)\right|\right) w^{\prime}(x)\right|^{p^{*}} d x\right)^{1 / p^{*}}\left\|v_{h}\right\|_{V_{h}} .
\end{aligned}
$$

Since the sequence $\left\{p_{h} v_{h}\right\}$ is weakly convergent in $V$ it is also bounded in $V$ such that $\left\|v_{h}\right\|_{V_{h}}=\left\|p_{h} v_{h}\right\|_{V} \leq c$ for some $c>0$. It remains to analyse the integral on the right-hand side of the foregoing estimate. This integral, however, converges towards zero, which follows from the continuity of $\psi$, the continuity of the Nemyzkii operator corresponding to $\psi$ as a mapping from $L^{p}(0,1)$ into $L^{p^{*}}(0,1)$ (see $[19$, Prop. 26.6 on p. 561] and remember the growth condition for $\psi$ ) as well as $p_{h} r_{h} w \rightarrow w$ in $V=W_{0}^{1, p}(0,1)$, i.e. $\left(p_{h} r_{h} w\right)^{\prime} \rightarrow w^{\prime}$ in $L^{p}(0,1)$.

For the second term $a_{2, h}$ in (7), we immediately have (in view of $p_{h} v_{h} \rightarrow v$ in $V$ )

$$
a_{2, h}=\left\langle A w, p_{h} v_{h}-v\right\rangle \rightarrow 0
$$

With respect to the right-hand side, we have to be somewhat careful as $f \in$ $L^{p^{*}}(0,1)$ does not allow to take point values. Instead, we may take

$$
f_{h, i}=\frac{1}{h} \int_{x_{i}}^{x_{i+1}} f(x) d x, \quad i=1, \ldots, M-1 .
$$


One can easily prove that $f_{h}$ is in $V_{h}^{*}$. Moreover, for arbitrary $v_{h} \in V_{h}$, we have

$$
\left\langle f_{h}, v_{h}\right\rangle=\sum_{i=1}^{M-1} \int_{x_{i}}^{x_{i+1}} f(x) d x v_{h, i}=h \sum_{i=1}^{M} \int_{x_{i}}^{1} f(x) d x D_{i}^{-} v_{h}
$$

and thus (with $1 / p+1 / p^{*}=1$ )

$$
\left\|f_{h}\right\|_{V_{h}^{*}}=\sup _{v_{h} \in V_{h} \backslash\{0\}} \frac{\left\langle f_{h}, v_{h}\right\rangle}{\left\|v_{h}\right\|_{V_{h}}} \leq\left(h \sum_{i=1}^{M}\left|\int_{x_{i}}^{1} f(x) d x\right|^{p^{*}}\right)^{1 / p^{*}} \leq\|f\|_{L^{p^{*}}(0,1)},
$$

which shows the boundedness of the sequence $\left\{\left\|f_{h}\right\|_{V_{h}}\right\}$ as required in Theorem 3.1. It remains to prove condition (iii) in Definition 2.2. Let $p_{h} v_{h} \rightarrow v$ in $V$, which implies that the sequence $\left\{p_{h} v_{h}\right\}$ is bounded in $V$. Since

$$
\begin{aligned}
\left\langle f_{h}, v_{h}\right\rangle-\left\langle f, p_{h} v_{h}\right\rangle & =\sum_{i=1}^{M-1} \int_{x_{i-1}}^{x_{i}} f(x)\left(v_{h, i}-\left(p_{h} v_{h}\right)(x)\right) d x \\
& =\sum_{i=1}^{M-1} \int_{x_{i-1}}^{x_{i}} f(x)\left(p_{h} v_{h}\right)^{\prime}(x)\left(x_{i}-x\right) d x
\end{aligned}
$$

and thus

$$
\left|\left\langle f_{h}, v_{h}\right\rangle-\left\langle f, p_{h} v_{h}\right\rangle\right| \leq h \int_{0}^{1}\left|f(x)\left(p_{h} v_{h}\right)^{\prime}(x)\right| d x \leq h\|f\|_{L^{p^{*}}(0,1)}\left\|p_{h} v_{h}\right\|_{V} \rightarrow 0,
$$

we obtain

$$
\left\langle f_{h}, v_{h}\right\rangle-\langle f, v\rangle=\left\langle f_{h}, v_{h}\right\rangle-\left\langle f, p_{h} v_{h}\right\rangle+\left\langle f, p_{h} v_{h}-v\right\rangle \rightarrow 0
$$

After all, Proposition 2.4 and Theorem 3.1 can be applied. This shows the existence of a weak solution $u \in V=W_{0}^{1, p}(0,1)$ to $(6)$ and a subsequence, denoted by $h^{\prime}$, such that the piecewise linear interpolations of the discrete solutions $u_{h^{\prime}}$ converge weakly in $V$ towards $u$.

\section{References}

[1] P.M. Anselone and J.-G. Lei, The approximate solution of monotone nonlinear operator equations, Rocky Mt. J. Math. 16 (1986) 4, 791-801.

[2] P.M. Anselone and J.-G. Lei, Nonlinear operator approximation theory based on demiregular convergence, Acta Math. Sci. 6 (1986), 121-132.

[3] J.-P. Aubin, Approximation des espaces de distributions et des opérateurs différentiels. Mémoires de la S.M.F. 12 (1967), 3-139.

[4] H. Brézis, Analyse fonctionnelle: Théorie et applications, Dunod, Paris, 1999.

[5] C. Davini and R. Paroni, External approximation of first order variational problems via $W^{-1, p}$ estimates, ESAIM Control Optim. Calc. Var. 14 (2008) 4, 802-824.

[6] H. Gajewski, K. Gröger and K. Zacharias, Nichtlineare Operatorgleichungen und Operatordifferentialgleichungen, Akademie-Verlag, Berlin, 1974.

[7] R.D. Grigorieff, Über diskrete Approximationen nichtlinearer Gleichungen 1. Art, Math. Nachr. 69 (1975) 253-272.

[8] J.-L. Lions, Quelques méthodes de résolution des problèmes aux limites non linéaires, Dunod, Gauthier-Villars, Paris, 1969. 
[9] W.V. Petryshin, Approximation-solvability of nonlinear functional and differential equations, M. Dekker, New York, 1993.

[10] T. Regińska, External approximation of eigenvalue problems in Banach spaces, RAIRO Anal. Numér. 18 (1984), 161-174.

[11] T. Regińska, Superconvergence of external approximation of eigenvalues of ordinary differential operators, IMA J. Numer. Anal. 6 (1986) 3, 309-323.

[12] T. Regińska, Superconvergence of external approximation for two-point boundary problems, Apl. Mat. 32 (1987) 1, 25-36.

[13] R. Schumann and E. Zeidler, The finite difference method for quasilinear elliptic equations of order 2m, Numer. Funct. Anal. Optimization 1 (1979), 161-194.

[14] F. Stummel, Diskrete Konvergenz linearer Operatoren I, Math. Ann. 190 (1970) 4592.

[15] R. Temam, Navier-Stokes equations: Theory and numerical analysis, AMS Chelsea Publ., American Mathematical Society, Providence, Rhode Island, 2001.

[16] R. Temam, Numerical analysis, D. Reidel Publ. Company, Dordrecht, 1973.

[17] R.U. Verma, On the external approximation-solvability of nonlinear equations, Panam. Math. J. 2 (1992) 3, 23-42.

[18] R.U. Verma, Stable discretization methods with external approximation schemes, $J$. Appl. Math. Stochastic Anal. 8 (1995) 4, 405-413.

[19] E. Zeidler, Nonlinear functional analysis and its applications, II/A: Linear monotone operators, II/B: Nonlinear monotone operators, Springer, New York, 1990. 